\title{
Predicting Individual Prognosis and Grade of Patients with Glioma Based on Preoperative Eosinophil and Neutrophil-to-Lymphocyte Ratio
}

This article was published in the following Dove Press journal: Cancer Management and Research

\author{
Xu Zhang $\mathbb{1}^{1,2}$ \\ Can $\mathrm{Li}^{1,2}$ \\ Lifei Xiao ${ }^{1,2}$ \\ Caibin Gao ${ }^{1,2}$ \\ Wei Zhao ${ }^{1,2}$ \\ Maolin Yang ${ }^{1,2}$ \\ Tao Sun ${ }^{1,2}$ \\ Feng Wang (1D ${ }^{1,2}$
}

'Department of Neurosurgery, General Hospital of Ningxia Medical University, Yinchuan 750000, People's Republic of China; ${ }^{2}$ Ningxia Key Laboratory of Cerebrocranial Disease, Incubation Base of National Key Laboratory, Ningxia Medical University, Yinchuan 750000,

People's Republic of China
Correspondence: Tao Sun; Feng Wang Tel +86 I389567099 I Email suntao_nxmu@163.com; nxwwang@163.com
Purpose: Eosinophils are proven to play a role in the prognosis of some malignant-tumors. The prognostic value of eosinophils in glioma patients is, however, scarcely reported. The authors of this article have designed a novel prognostic indicator based on eosinophils and the neutrophil-to-lymphocyte ratio (NLR), named ENS, to predict the survival of patients with glioma.

Methods: A retrospective study was conducted on 217 glioma patients. The cut-off values for eosinophil, NLR, and other clinical variables were determined by the receiver operating characteristic (ROC) curve analysis. Patients with both low eosinophil count $\left(<0.08 \times 10^{9} / \mathrm{L}\right)$ and high NLR $(\geq 1.70)$ were given a score of 2 . Those with one or neither got a score of 1 or 0 , respectively. The nomogram was based on ENS and several other clinical variables, its performance was determined by the concordance index (c-index).

Results: Our results showed that ENS is an independent prognostic indicator for overall survival (OS). The three-year OS rates for low-grade glioma patients (LGGs) were $84.0 \%$, $69.0 \%$, and $46.4 \%$ for $\mathrm{ENS}=0$, ENS $=1$, and $\mathrm{ENS}=2$, respectively $(P=0.014)$. The three-year OS incidence for LGGs stratified into eosinophils count $\geq 0.08 \times 109 / \mathrm{L}$ and $<0.08 \times 109 / \mathrm{L}$ subgroups were $88.1 \%$ and $80.0 \%$, respectively $(P=0.043$ ). ENS was positively correlated with glioma grade $(\mathrm{r}=0.311, P<0.001)$. The $\mathrm{c}$-index for OS prognosis was $0.80 \mathrm{using}$ this nomogram in LGGs.

Conclusion: Preoperative ENS can predict OS to some extent for LGGs and can increase prognostic accuracy for individual OS in LGGs postoperatively when incorporating other clinical variables compose a nomogram.

Keywords: low-grade glioma, eosinophil, neutrophil-to-lymphocyte ratio, nomogram, prognosis

\section{Introduction}

Glioma is the most common primary intracerebral tumor. ${ }^{1}$ According to the World Health Organization (WHO) classification, it can be divided into four histopathological grades (ie, WHO grade I, II, III, and IV). ${ }^{2}$ We generally define WHO grade I-II tumor as low-grade glioma (LGG), and grade III-IV as high-grade glioma (HGG). Numerous studies have proved that glioma is associated with high recurrence rate and poor prognosis. ${ }^{3}$ Additionally, high-grade glioma patients have worse prognosis than low-grade glioma patients, especially for glioblastoma patients, in which people older than 65 years have a 2-year survival probability of less than $5 \%{ }^{4}$ The prognosis of glioma patients directly determines the quality of their life 
and treatment plans. ${ }^{5}$ At present, the WHO classification still plays an irreplaceable role in predicting the prognosis of gliomas. In recent years however, researchers have paid more attention to other auxiliary molecular predictive indicators, such as isocitrate dehydrogenase (IDH) and O-6-methylguanine-DNA-methyltransferase (MGMT). ${ }^{6-9}$ However, these indicators can only be obtained postoperatively. Preoperative peripheral blood examination; which has the merits of easy accessibility and low cost, predicts the prognosis of many tumors, such as lung cancer ${ }^{10}$ and Laryngeal squamous cell carcinoma. ${ }^{11}$ The peripheral blood biomarkers also play an essential role in the emergence and growth of glioma. For the past few years, there have been many reports of peripheral blood cells, such as lymphocytes and neutrophils, affecting the prognosis of gliomas. ${ }^{12,13}$

Eosinophils are innate immune cells that have outstanding performance in both atopic diseases and allergic reactions. They are also proven to be associated with the prognosis of some solid tumors. ${ }^{14,15}$ The relationship between eosinophils and glioma prognosis has however been scarcely reported. Some studies have combined biomarkers to improve the prognostic value of malignant tumors, such as F-NLR, which combines fibrinogen and neutrophil/lymphocyte ratio. ${ }^{16,17}$ Thus, we designed a novel prognostic indicator named ENS, which combines eosinophil and neutrophil/lymphocyte ratio to improve predictive power. Nomograms are universal in the field of oncology for calculating the individual prognosis of different cancers. This study introduces a novel scoring tool (ENS) that, when combined with other clinical factors, can compose a nomogram to predict the 3- and 5-year OS for postoperative glioma patients.

\section{Materials and Methods}

\section{Patients}

A total of 217 glioma patients from the Department of Neurosurgery, General Hospital of Ningxia Medical University were included in our study. These patients underwent surgery between January 2011 and March 2017 and had a histological diagnosis of glioma according to the WHO 2007 classification. The inclusion criteria were as follows: (1) Complete clinical data and routine blood examination before operation. (2) Gross total tumor resection. The exclusion criteria were as follows: (1) The tumor was a recurrent glioma. (2) Patients received chemoradiotherapy before surgery. (3) Patients received steroids and other immune modulating treatments. (4) Patients with hemopathy, infectious diseases, or immune system disorders. (5) The patients have other systemic tumors. (6) The patients died in the hospital during the perioperative period. (7) Patients declined followed up. According to the above criteria, we followed up patients by phone semiannually. The last follow-up date was on 1st, March, 2020. We defined OS as the duration of time from the date of reaching the definitive diagnosis to death.

We collected clinical parameters of patients including sex, age, tumor size, tumor WHO grade (I/II/III/IV), Karnofsky performance status (KPS), preoperative eosinophils count, neutrophil count, lymphocyte count, and postoperative adjuvant chemoradiotherapy.

\section{ENS Definition}

The NLR was defined by dividing neutrophil count by lymphocyte count. We used receiver operating characteristic (ROC) curve analysis to identify the optimal cut-off values for the preoperative eosinophils count and NLR. The cut-off values were $0.08 \times 10^{9}$ for eosinophils and 1.70 for NLR [sensitivity, specificity, and area under the curve (AUC): $59.6 \%, 59.2 \%$ and 0.629 for eosinophils; $70.9 \%$, $61.4 \%$ and 0.666 for NLR, respectively]; The ENS was then calculated as follows: patients with low eosinophils $\left(<0.08 \times 10^{9}\right)$ and high NLR $(\geq 1.70)$ were given a score of 2 (two abnormalities) if either one parameter reached previously mentioned standards they were defined as a score of 1 (one anomaly), and if no settings met the criteria they were defined as a score of 0 (no defect).

\section{Statistical Analysis}

The optimal cut-off values for eosinophil count and NLR were determined by receiver-operating characteristic (ROC) curve analysis. Unpaired $t$-test was used for comparison of the two groups. The correlation between ENS and WHO grade was analyzed by Spearman's rank correlation and Mann-Whitney rank-sum test. The relationship between the three ENS groups and other clinical variables of glioma patients was evaluated by the Chi-squared test. Categorical variables and continuous variables were shown as number and mean $\pm \mathrm{SD}$, respectively. Survival analyses were plotted using the Kaplan-Meier survival curve, and differences were evaluated by the Log rank test. The Cox regression model was used for performing the univariate and multivariate analyses of clinical variables to identify the independent prognostic factors. All 
reported $P$ values were two-sided, and $P<0.05$ was considered statistically significant. The above statistical analyses were performed with SPSS software (version 22.0) and GraphPad Prism (version 8.0). The nomogram was designed using $\mathrm{R}$ software (Version 3.6.3). The concordance index (c-index) was used to quantify the predictive performance of the nomogram.

\section{Results}

\section{Patient Clinical Characteristics}

Of the 217 patients, 116 (53.5\%) were male, and 101 (46.5\%) were female, ranging from 8 to 77 years old. Their mean age was $48 \pm 15.03$ years. The tumor size was $<5 \mathrm{~cm}$ in $114(53.0 \%)$ patients and $\geq 5 \mathrm{~cm}$ in $103(47.0 \%)$ patients. $28.1 \%$ of patients had a KPS $<70$ while 146 (71.9\%) of patients had a KPS $\geq 70$. Sixty-four percent of the patients received postoperative treatment, including radiotherapy or chemotherapy. The mean pretreatment eosinophil, neutrophil, and lymphocyte counts were 0.09 $\pm 0.07 \times 10^{9} / \mathrm{L}, \quad 3.95 \pm 2.02 \times 10^{9} / \mathrm{L}$, and $1.93 \pm 0.66 \times 109 / \mathrm{L}$, respectively. The mean pretreatment NLR was $2.34 \pm 1.98$. Approximately $27.2 \%$ of patients had ENS $0,42.4 \%$ had ENS 1 and 30.4\% had ENS 2 (Table 1).

\section{Correlations Between ENS and Clinical Parameters}

The decrease in eosinophil count (Figure 1A) and increase in neutrophil count are more significant (Figure 1B) in high-grade gliomas (WHO grade III+ IV) compared to low-grade gliomas (WHO grade I+ II). While we found no significant association between lymphocyte count and WHO grade (Figure 1C). The correlation between ENS and glioma grade was further evaluated, showing that ENS was positively correlated with glioma grade $(\mathrm{r}=0.311$, $P<0.001)$, and the high-grade group had higher ENS $(P<0.001)$ (Figure 1D).We also evaluated the association between ENS and other clinical parameters of patients with glioma, and we observed significant differences among the ENS 0, 1, 2 groups in terms of tumor size $(P=0.009)$, WHO grade $(P=0.009)$ and KPS score $(P=0.021)$. Nevertheless, no significant differences were found in the distribution of gender, age, and adjuvant chemoradiotherapy (Table 2).

\section{Prognostic Factors}

The age $(\mathrm{HR}=4.356 ; 95 \% \mathrm{CI}=2.826-6.714 ; P<0.001)$, tumor size $(\mathrm{HR}=1.748 ; 95 \% \mathrm{CI}=1.181-2.588 ; P=0.05)$, WHO
Table I Characteristics of Patients

\begin{tabular}{|l|l|l|}
\hline Variables & Value & Percentage $\%$ \\
\hline Sex & & \\
Male & 116 & $53.5 \%$ \\
Female & 101 & $46.5 \%$ \\
\hline Age $(y)$ & $48 \pm 15.03$ & \\
$<50$ & 115 & $53.0 \%$ \\
$\geq 50$ & 102 & $47.0 \%$ \\
\hline Tumor size $(\mathrm{cm})$ & $4.57 \pm 1.38$ & \\
$<5$ & 114 & $53.0 \%$ \\
$\geq 5$ & 103 & $47.0 \%$ \\
\hline WHO Grade & & \\
I + II & 128 & $59.0 \%$ \\
III + IV & 89 & $41.0 \%$ \\
\hline KPS & $67.24 \pm 10.87$ & \\
$<70$ & 61 & $28.1 \%$ \\
$\geq 70$ & 146 & $71.9 \%$ \\
\hline Radiotherapy/Chemotherapy & & NA \\
Yes & 138 & $64.0 \%$ \\
No & 79 & $36.0 \%$ \\
\hline Eosinophils $\left(\times 10^{9}\right)$ & $0.09 \pm 0.07$ & NA \\
Neutrophil $\left(\times 10^{9}\right)$ & $3.95 \pm 2.02$ & $30.4 \%$ \\
Lymphocyte $\left(\times 10^{9}\right)$ & $1.93 \pm 0.66$ & NA \\
NLR & $2.34 \pm 1.98$ & \\
\hline ENS & 56 & \\
0 & 59 & \\
I & & \\
2 & NA & \\
\hline
\end{tabular}

Abbreviations: KPS, Karnofsky performance status; NLR, neutrophil to lymphocyte ratio; ENS, the combination of eosinophils, and neutrophil to lymphocyte ratio.

grade $(\mathrm{HR}=6.175 ; 95 \% \mathrm{CI}=4.070-9.367 ; P<0.001)$, KPS $(\mathrm{HR}=0.544 ; 95 \% \mathrm{CI}=0.364-0.814 ; P=0.003)$ and ENS $(P<0.001)$ were closely associated with poor OS in the univariate analysis. In addition to this, multivariate analysis indicated that age $(\mathrm{HR}=4.296 ; 95 \% \mathrm{CI}=2.748-6.716$; $P<0.001)$, WHO grade $(\mathrm{HR}=5.951 ; 95 \% \mathrm{CI}=3.694-9.588$; $P<0.001)$, KPS (HR $=0.436 ; 95 \% \mathrm{CI}=0.281-0.675 ; P<0.001)$ and ENS $(P=0.047)$ were independent factors of OS, respectively (Table 3 ).

In order to eliminate the bias caused by WHO grade as much as possible, we analyzed the relationship between eosinophils, NLR, ENS and OS based on low-grade gliomas and high-grade gliomas. The OS probability analysis indicated that the three-year OS incidence for LGGs stratified into eosinophils count $\geq 0.08 \times 10^{9} / \mathrm{L}$ and $<0.08 \times 10^{9} / \mathrm{L}$ subgroups were $88.1 \%$ and $80.0 \%$, respectively. These 


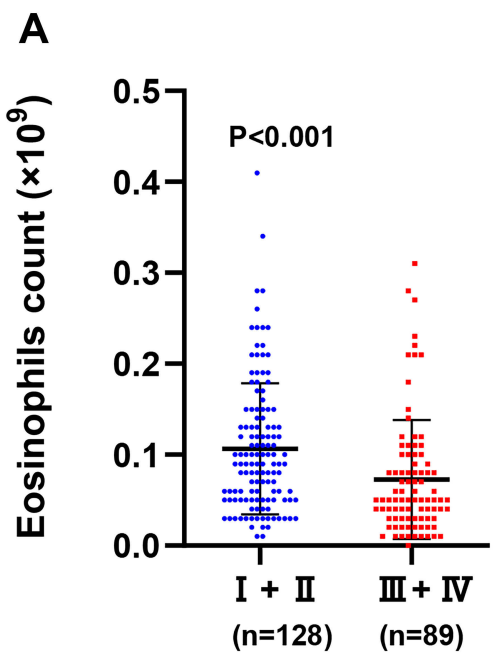

B

C

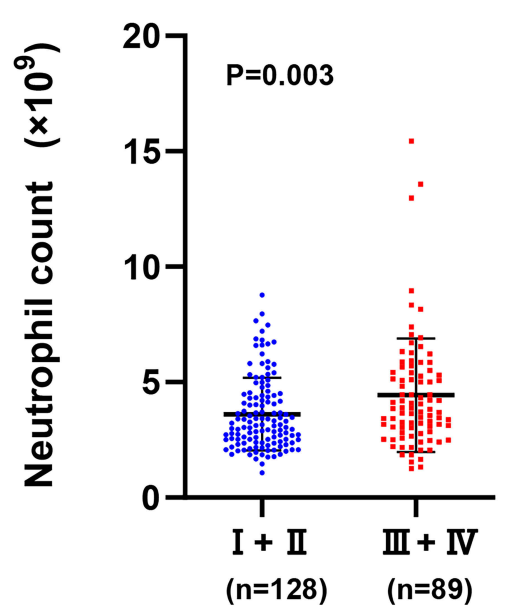

D
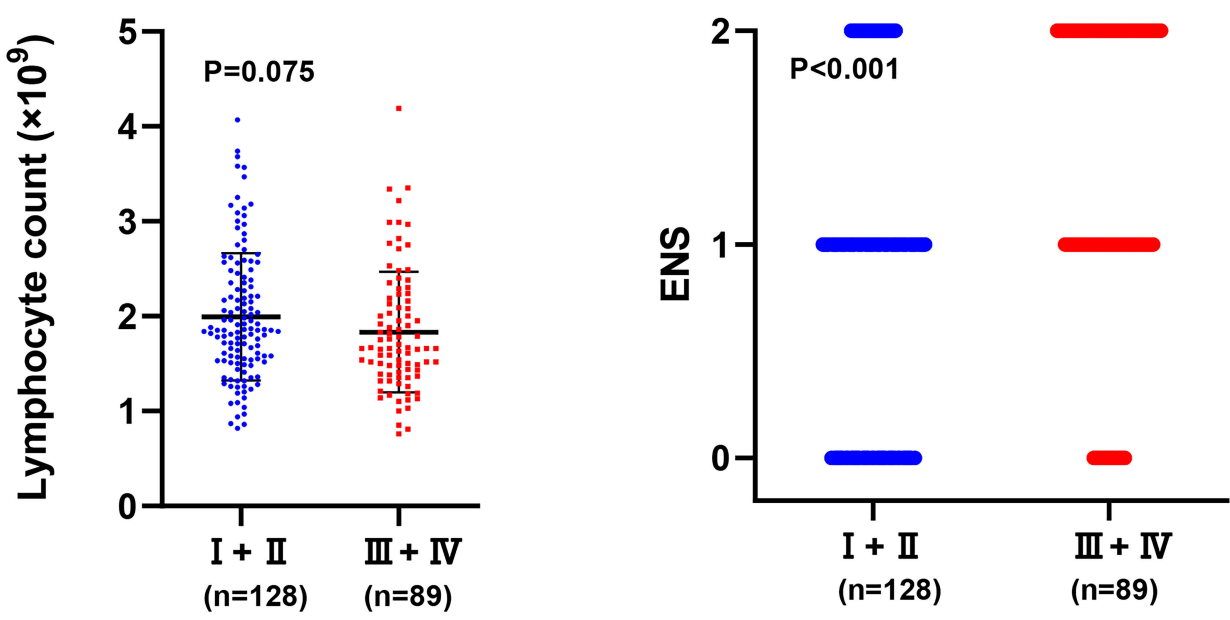

Figure I The correlations between eosinophil, neutrophil, lymphocyte count, ENS, and glioma grade. (A) The diversity of eosinophil count in different glioma grades. (B) The variety of neutrophil count in different glioma grades. (C) Distribution of lymphocyte count in low-grade and high-grade glioma patients. (D) Distribution of ENS in low and high-grade glioma patients.

differences in OS were significant according to the Log rank test $(P=0.043$; Figure $2 \mathrm{~A})$, while there was no statistical significance in high-grade glioma patients (HGGs) (Figure 2B). The OS probability analysis manifested that the three-year OS incidence for LGGs divided into NLR $<1.70$ and $\geq 1.70$ subgroups were $91.5 \%$ and $75.8 \%$, respectively ( $P=0.015$; Figure $2 \mathrm{C}$ ), and the three-year OS incidence for HGGs were $38.1 \%$ and $16.8 \%$, respectively $(P=0.026$; Figure 2D). We also use Kaplan-Meier analysis to determine the survival differences among the three groups divided by ENS, the three-year OS rate in LGGs with ENS 2 were lower than those of patients with ENS $=1$ or $0[72.0 \%$ vs $83.3 \%$, or $93.5 \%, P=0.014$ (Figure $2 \mathrm{E}$ )], however there was no statistical significance in HGGs (Figure 2F).

\section{Nomogram for LGGs}

The prognostic nomogram for speculating individual 3-year and 5-year OS for LGGs is showed in Figure 3A. The c-index for the nomogram was 0.80 ( $95 \% \mathrm{CI}, 0.785$ to $0.855)$. The results indicated that the signature age contributed the most risk points (range, 0-100). Nevertheless, other clinical variables, including KPS score and ENS, exhibited smaller contributions. We also found that the calibration curve performed excellent agreement between prediction and observation in predicting 3-, and 5-year overall survival (Figure 3B and C).

\section{Discussion}

In our study, we combined eosinophil count and NLR, named ENS, as a forecasting tool. We found that decreased 
Table 2 Association Between ENS and Clinicopathological Data of Patients

\begin{tabular}{|c|c|c|c|c|}
\hline Variables & $\begin{array}{l}E N S=0 \\
(n=59)\end{array}$ & $\begin{array}{l}E N S=1 \\
(n=92)\end{array}$ & $\begin{array}{l}\text { ENS }=2 \\
(n=66)\end{array}$ & $P$ value \\
\hline \multicolumn{5}{|l|}{ Sex } \\
\hline Male & $34(57.6 \%)$ & 43 (46.7\%) & $39(59.1 \%)$ & 0.232 \\
\hline Female & $25(42.4 \%)$ & 49 (53.3\%) & $27(40.9 \%)$ & \\
\hline \multicolumn{5}{|l|}{ Age $(y)$} \\
\hline$<50$ & 35 (59.3\%) & 49 (53.3\%) & 31 (47.0\%) & 0.384 \\
\hline$\geq 50$ & $24(40.7 \%)$ & 43 (46.7\%) & 35 (53.0\%) & \\
\hline \multicolumn{5}{|l|}{ Tumor size $(\mathrm{cm})$} \\
\hline$<5$ & 38 (64.4\%) & $5 \mathrm{I}(55.4 \%)$ & 25 (37.9\%) & 0.009 \\
\hline$\geq 5$ & 21 (35.6\%) & 41 (44.6\%) & 41 (62.1\%) & \\
\hline \multicolumn{5}{|l|}{ WHO Grade } \\
\hline $1+\|$ & 46 (78.0\%) & 57 (62.0\%) & 25 (37.9\%) & 0.009 \\
\hline III + IV & $13(22.0 \%)$ & 35 (38.0\%) & $41(62.1 \%)$ & \\
\hline \multicolumn{5}{|l|}{ KPS } \\
\hline$<70$ & 14 (23.7\%) & 20 (21.7\%) & 27 (40.9\%) & 0.021 \\
\hline$\geq 70$ & $45(76.3 \%)$ & 72 (78.3\%) & $39(59.1 \%)$ & \\
\hline \multicolumn{5}{|l|}{ Radiotherapy/ } \\
\hline \multicolumn{5}{|l|}{ Chemotherapy } \\
\hline Yes & 37 (62.7\%) & 57 (62.0\%) & 44 (66.7\%) & 0.821 \\
\hline No & $22(37.3 \%)$ & 35 (38.0\%) & $22(33.3 \%)$ & \\
\hline
\end{tabular}

Abbreviations: KPS, Karnofsky performance status; ENS, the combination of eosinophils, and neutrophil to lymphocyte ratio.

eosinophil count, higher NLR and rising ENS score before surgery were negative predictors of OS for low-grade glioma patients, which indicated that the ENS may be a significant indicator for LGGs. We also designed a nomogram that includes the ENS and other clinical parameters to improve prognosis prediction in patients of low-grade glioma. Eosinophil count and ENS score, however, had no prognostic value in HGGs. This may need further study for there were only $89 \mathrm{HGGs}$ in our study.

Eosinophil, an innate immune cell, has been reported as a vital regulator of both atopic diseases and allergic reactions. Besides, it has close relations with many intricate immunoreactions, including tumorigenesis. However, the functions that eosinophils perform in tumorigenesis are still in dispute. There is no doubt, however, that eosinophils can infiltrate tumors and are associated with a favorable prognosis in most cases, ${ }^{18}$ such as nasopharyngeal cancer $^{14}$ and oral squamous cell carcinoma. ${ }^{15}$ Interestingly, from the epidemiological perspective, glioma risk has consistently been inversely associated with the patients who suffer allergic diseases and asthma. ${ }^{19-21}$ And they often have high-level eosinophils.
This phenomenon shows that eosinophils may inhibit the progress of glioma growth. However, the mechanisms of interaction between eosinophils and tumor tissue remain elusive. A recent study shows that eosinophils cluster near and within tumor tissue. ${ }^{22}$ This phenomenon is called tumor-associated tissue eosinophilia (TATE), it often represents a favorable prognosis. ${ }^{18}$ A study by Simson et $\mathrm{al}^{23}$ shows that tumor incidence and growth is significantly suppressed in mice that have elevated levels of circulating eosinophils compared with those with low levels of circulating eosinophils. They also found that eosinophils directly kill methylcholanthrene (MCA)induced fibrosarcoma cells in subsequent vitro studies. Although the mechanisms between eosinophils and tumor suppression are not entirely clear, numerous studies have proved that eosinophils affect prognosis in some malignancies. But we find no clinical research investigating the prognosis of glioma based on eosinophils. Our study demonstrates that LGGs with low preoperative eosinophil count had shorter OS than those with high preoperative eosinophil count. We also discovered the fact that the level of eosinophil count has a statistic difference between lowand high-grade glioma (Figure 1A), which is also in line with Huang et al. ${ }^{24}$ Therefore, preoperative eosinophils count can evaluate the prognosis of LGGs to some extent.

Recently, numerous studies have proved that inflammatory biomarkers play an important role in the prognosis of malignant tumors. Several prognostic biomarkers based on blood cells, relating to systemic inflammation responses, were created to predict patient outcome. These included neutrophil/lymphocyte ratio (NLR), platelet/lymphocyte ratio (PLR), lymphocyte/macrophage ratio (LMR) and so on. Several pieces of research have proved that an elevated NLR indicates a poor prognosis in some solid tumors. ${ }^{11,25,26}$ Neutrophils have been proved to contribute to tumor angiogenesis, depletion of these neutrophils could therefore inhibit tumor growth. ${ }^{27}$ On the other hand, tumors infiltrated by numerous lymphocytes have been associated with excellent prognosis in several cancers. $^{28,29}$ Like other solid tumors, NLR could be used as a prognostic tool to predict the prognosis of glioma. ${ }^{30}$ An increased peripheral blood neutrophil/lymphocyte ratio (NLR) is associated with poor prognosis in glioma. ${ }^{12,13,31}$ Our study demonstrated that elevated NLR was associated with poor OS in both low- and high-grade glioma patients. In line with Huang et al, ${ }^{24}$ we also found that a rising trend of neutrophil counts between low-grade glioma and highgrade glioma (Figure 1B). 
Table 3 The Univariate and Multivariate Analysis of OS in Glioma Patients

\begin{tabular}{|c|c|c|c|c|}
\hline \multirow[t]{2}{*}{ Variables } & \multicolumn{2}{|l|}{ Univariate Analysis } & \multicolumn{2}{|c|}{ Multivariate Analysis } \\
\hline & HR (95\% Cl) & $\mathbf{P}$ & HR $(95 \% \mathrm{Cl})$ & $\mathbf{P}$ \\
\hline \multicolumn{5}{|l|}{ Sex } \\
\hline Male & Reference & 0.277 & Reference & 0.296 \\
\hline Female & $0.806(0.546-1.190)$ & & $0.804(0.534-1.211)$ & \\
\hline \multicolumn{5}{|l|}{ Age $(y)$} \\
\hline$<50$ & Reference & $<0.001$ & Reference & $<0.001$ \\
\hline$\geq 50$ & $4.356(2.826-6.714)$ & & $4.296(2.748-6.716)$ & \\
\hline \multicolumn{5}{|c|}{ Tumor size $(\mathrm{cm})$} \\
\hline$<5$ & Reference & 0.005 & Reference & 0.702 \\
\hline$\geq 5$ & $1.748(1.181-2.588)$ & & $0.922(0.609-1.396)$ & \\
\hline \multicolumn{5}{|c|}{ WHO Grade } \\
\hline $1+I I$ & Reference & $<0.001$ & Reference & $<0.001$ \\
\hline III + IV & $6.175(4.070-9.367)$ & & 5.951 (3.694-9.588) & \\
\hline \multicolumn{5}{|l|}{ KPS } \\
\hline$<70$ & Reference & 0.003 & Reference & $<0.001$ \\
\hline$\geq 70$ & $0.544(0.364-0.814)$ & & $0.436(0.28 I-0.675)$ & \\
\hline \multicolumn{5}{|c|}{ Radiotherapy/Chemotherapy } \\
\hline Yes & Reference & 0.994 & Reference & 0.885 \\
\hline No & 1.001 (0.669-I.499) & & 1.031 (0.683-I.557) & \\
\hline \multicolumn{5}{|l|}{ ENS } \\
\hline Score $=0$ & Reference & $<0.001$ & Reference & 0.047 \\
\hline Score $=1$ & $1.902(1.064-3.400)$ & 0.030 & $1.572(0.870-2.84 I)$ & 0.134 \\
\hline Score $=2$ & $3.843(2.175-6.79 \mid)$ & $<0.001$ & $2.152(1.163-3.983)$ & 0.015 \\
\hline
\end{tabular}

Abbreviations: KPS, Karnofsky performance status; ENS, the combination of eosinophils, and neutrophil to lymphocyte ratio; HR, hazard ratio; Cl, confidence interval.

We developed a new prognostic score; ENS, which combines eosinophil count and NLR. It is the first time establishing a scoring tool to evaluate the prognosis of glioma patients based on eosinophils. The novel ENS has significant prognostic potential for LGGs. However, it's worth noting that despite the whole scoring system had a $P$-value of $<0.05$, ENS $=1$ was not statistically significant (Table 3 ). This probably because we used ENS $=0$ as the reference or the prognostic difference between $\mathrm{ENS}=0$ and 1 was not significant enough. The correlation between ENS and glioma grade was also further evaluated, indicating that ENS was positively correlated with glioma grade. In the subgroup of high-grade gliomas (WHO grade III + IV), the ENS was significantly higher than those with lowgrade (WHO grade I + II) gliomas. The ENS may, therefore, be an auxiliary tool to predict the degree of glioma in the future, as it can easily be obtained by routine blood examination.

Nomograms are widely used to determine the prognosis of tumors, mainly because of their ability to generate a personal probability of a clinical event by incorporating diverse determinant variables. ${ }^{32,33}$ They can be tools to predict patient prognosis in addition to WHO grade, which is the current gold standard. They have been used in several malignant tumors as adjuncts in prognostic determination. ${ }^{34,35} \mathrm{In}$ our predictive nomogram, variables such as age and KPS have also been proved to be associated with long-term survival in other studies. ${ }^{36,37}$ Besides, the current nomogram first incorporates ENS, which has been verified as an independent factor assessing prognosis in our study. The c-index for the nomogram was 0.80 , and the calibration curve performed perfect agreement between prediction and observation in predicting 3-, and 5-year overall survival.

Our current study has several limitations. First, this study may introduce selection bias due to its retrospective and single-center nature. Thus a prospective, large-sample and multi-center research is needed. Second, the cut-off values for eosinophil count and NLR were 0.08 and 1.70 for this specific study population. However, when reviewing the literature, these biomarkers have different cut-off 
A

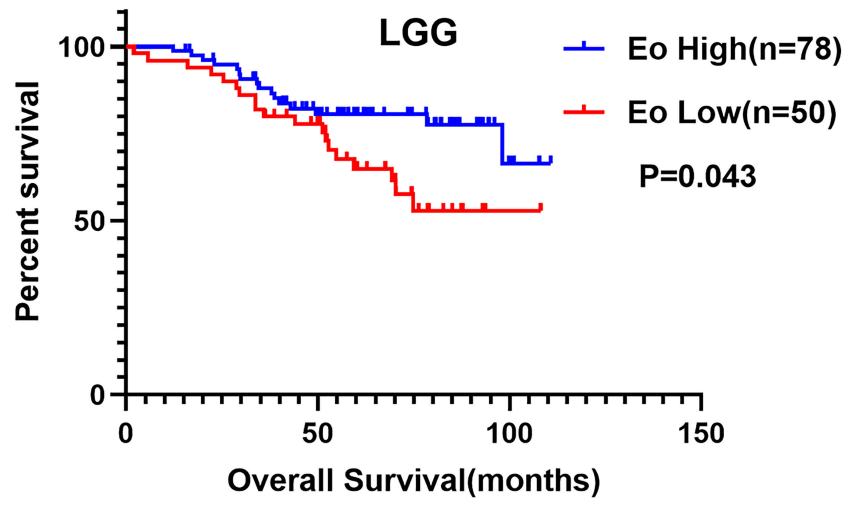

C

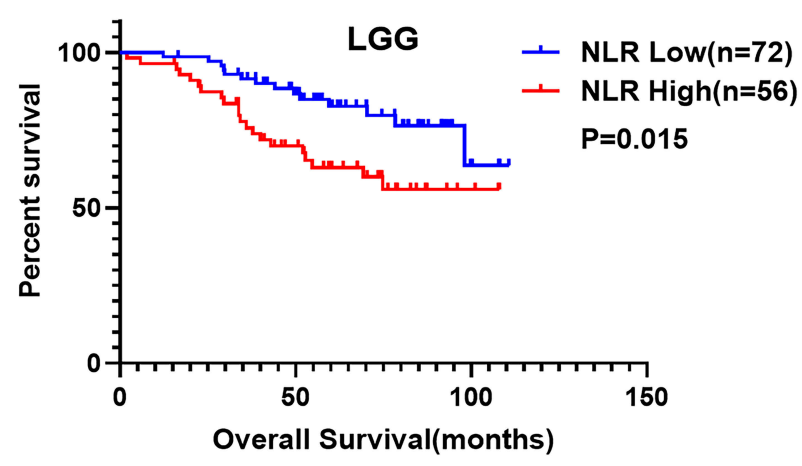

E

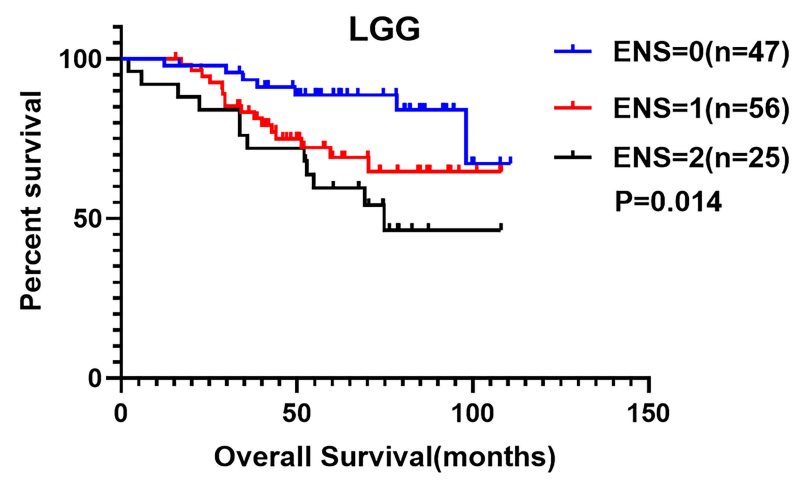

B

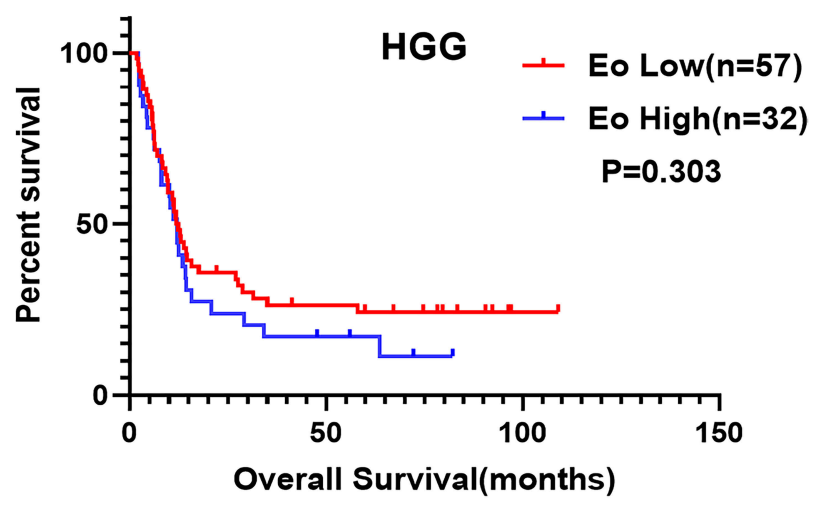

D

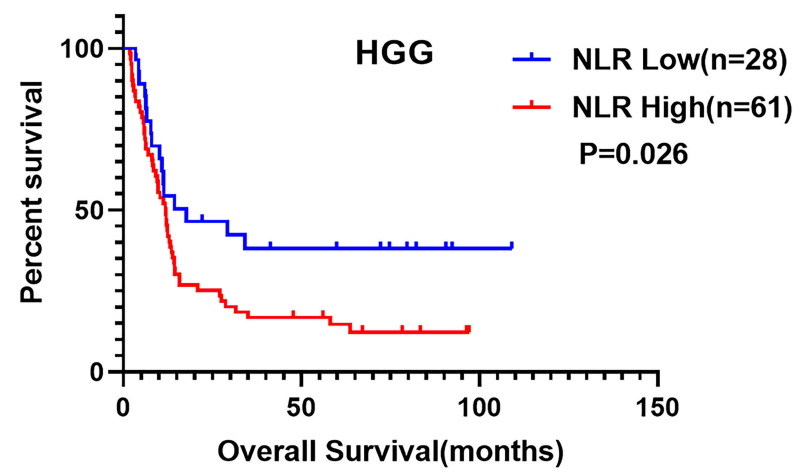

F

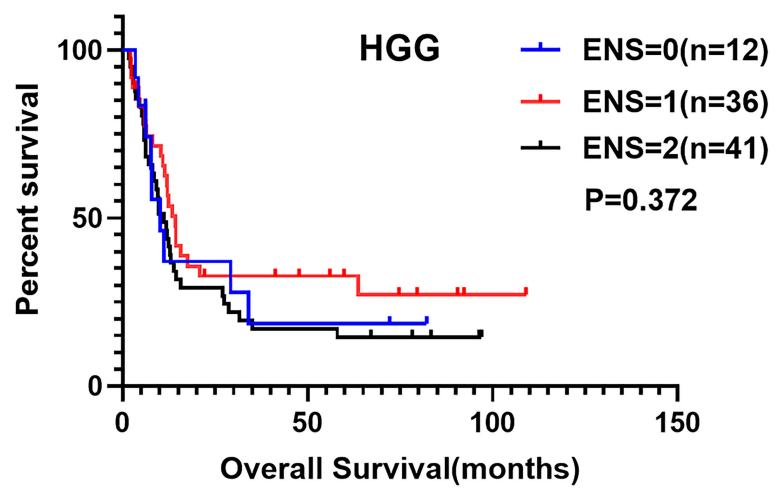

Figure 2 Kaplan-Meier survival curves for OS (overall survival) according to preoperative (A) Eo (eosinophils), (C) NLR, (E) ENS in patients with low-grade glioma. KaplanMeier survival curves for OS according to preoperative (B) Eo (eosinophils), (D) NLR, (F) ENS in patients with high-grade glioma.

values based on cancer type and study group. ${ }^{24,35,38}$ Thus it is hard to create standardized cut-offs that can be used around the world, even within a single tumor type. Third, biomarkers such as IDH status, MGMT or other genetic indicators were not included in our study owing to the immunohistochemical analysis results and frozen specimens were incomplete in several early cases. Finally, though the nomogram internally validated was perfect, external validation is necessary to affirm whether our findings are universally applicable. 
A

Points

$\begin{array}{lllllllllll}0 & 10 & 20 & 30 & 40 & 50 & 60 & 70 & 80 & 90 & 100\end{array}$

age

$\begin{array}{lllllllllllllll}75 & 70 & 65 & 60 & 55 & 50 & 45 & 40 & 35 & 30 & 25 & 20 & 15 & 10 & 5\end{array}$

KPS

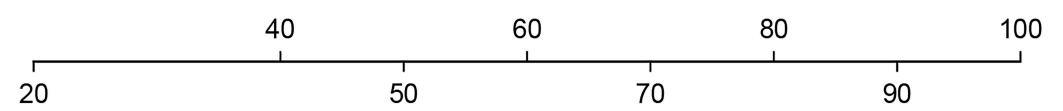

ENS

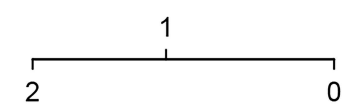

Total Points

\begin{tabular}{lllllllllll}
\hline & 20 & 40 & 60 & 80 & 100 & 120 & 140 & 160 & 180 & 200
\end{tabular}

3-year Survival Probability

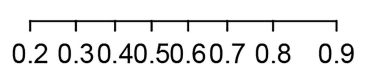

5-year Survival Probability

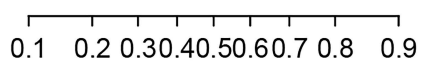

B

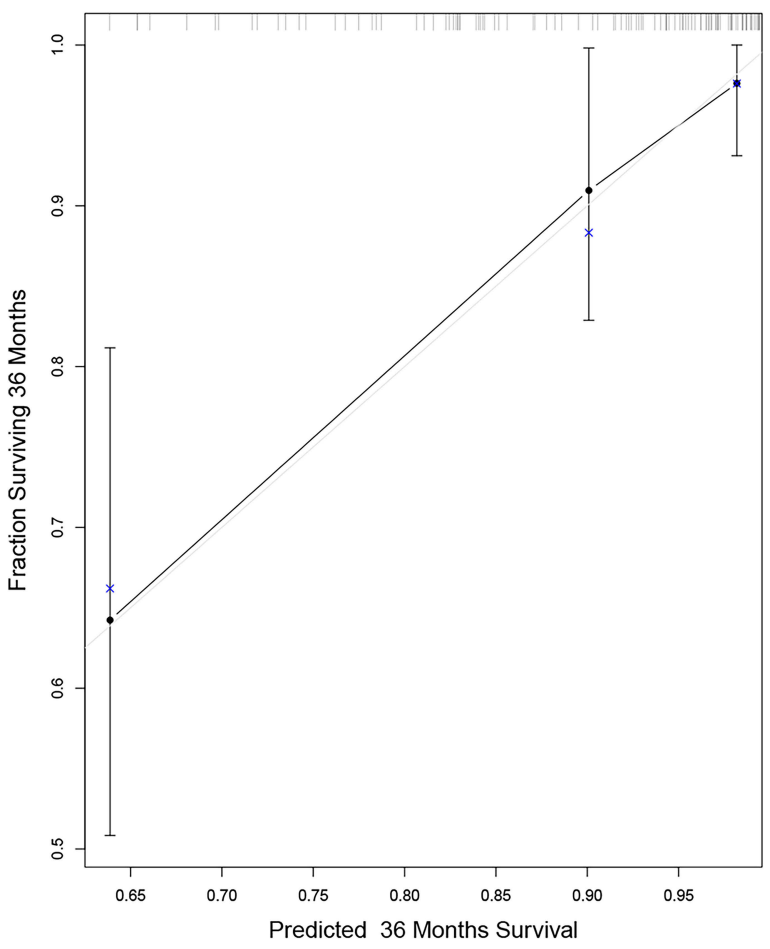

C

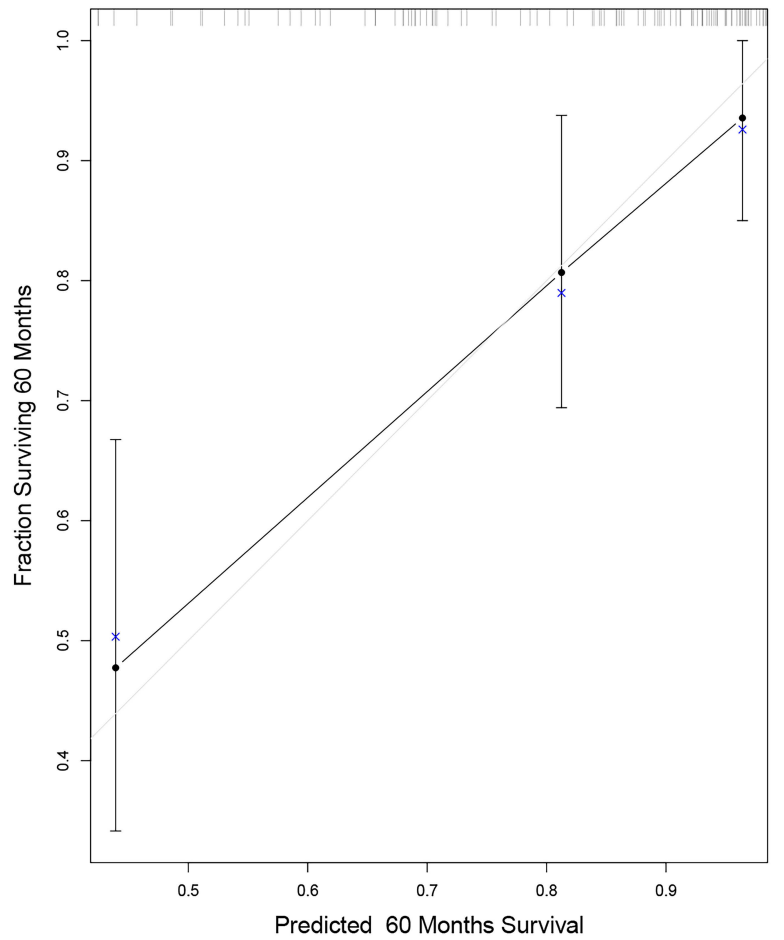

Figure 3 Nomogram and calibration curve. (A) Nomogram for OS prediction of low-grade glioma patients. Drawing a vertical line from each factor to the point score. A total points score is calculated when adding the points from all elements, drawing a vertical line to its axis, the 3-year and 5-year OS probabilities could be known. (B) The calibration curve to predict 3-year OS. The gray line indicates the ideal prediction, and the black line represents the performance of the nomogram. (C) The calibration curve for 5 -year OS prediction. 


\section{Conclusion}

The new scoring tool ENS, can potentially be a new preoperative assessment tool to predict the grade of glioma patients. It's also a prognostic assessment tool for LGGs.

A nomogram was established by combining the ENS and other clinical variables. This nomogram might provide more individual and accurate prognostic information for clinicians and low-grade glioma patients.

\section{Abbreviations}

NLR, neutrophil-to-lymphocyte ratio; ENS, the combination of eosinophils, and neutrophil to lymphocyte ratio; LGGs, low-grade glioma patients; Eo, eosinophil; OS, overall survival; ROC, receiver operating characteristic; AUC, area under the curve; CI, confidence interval; KPS, Karnofsky performance status; HR, hazard ratio.

\section{Data Sharing Statement}

Materials described in the manuscript, including all relevant raw data, will be freely available to any scientist wishing to use them for non-commercial purposes.

\section{Ethics Approval and Informed Consent}

Patient data confidentiality was maintained, in accordance with the Declaration of Helsinki. Ethical approval was waived by the local Ethics Committee of Ningxia Medical University in view of the retrospective nature of the study and all the procedures being performed were part of the routine care. Informed consent was obtained from all individual participants included in the study. A parent or legal guardian provided informed consent for any patient under the age of 18 years.

\section{Acknowledgment}

The authors thank the help of Record room of General Hospital of Ningxia Medical University.

\section{Author Contributions}

All authors made substantial contributions to conception and design, acquisition of data, or analysis and interpretation of data; took part in drafting the article or revising it critically for important intellectual content; gave final approval of the version to be published; and agree to be accountable for all aspects of the work.

\section{Funding}

This work was supported by grants from the Key Research and Development Program of Ningxia Hui Autonomous Region (2018BFG02007).

\section{Disclosure}

The authors declare that they have no competing interests.

\section{References}

1. Rasmussen BK, Hansen S, Laursen RJ, et al. Epidemiology of glioma: clinical characteristics, symptoms, and predictors of glioma patients grade I-IV in the the Danish Neuro-Oncology Registry. J Neurooncol. 2017;135(3):571-579. doi:10.1007/ s11060-017-2607-5

2. Louis DN, Ohgaki H, Wiestler OD, et al. The 2007 WHO classification of tumours of the central nervous system. Acta Neuropathol. 2007;114(2):97-109. doi:10.1007/s00401-007-0243-4

3. Bie L, Zhao G, Cheng P, et al. The accuracy of survival time prediction for patients with glioma is improved by measuring mitotic spindle checkpoint gene expression. PLoS One. 2011;6(10):e25631. doi:10.1371/journal.pone.0025631

4. Anton K, Baehring JM, Mayer T. Glioblastoma multiforme: overview of current treatment and future perspectives. Hematol Oncol Clin North Am. 2012;26(4):825-853. doi:10.1016/j.hoc.2012.04.006

5. Nabors LB, Portnow J, Ammirati M, et al. NCCN guidelines insights: central nervous system cancers, version 1. 2017. J Natl Compr Canc Netw. 2017;15(11):1331-1345. doi:10.6004/jncen.2017.0166

6. Leu S, von Felten S, Frank S, et al. IDH/MGMT-driven molecular classification of low-grade glioma is a strong predictor for long-term survival. Neuro-Oncology. 2013;15(4):469-479. doi:10.1093/neuonc/nos317

7. Koh J, Cho H, Kim H, et al. IDH2mutation in gliomas including novel mutation. Neuropathology. 2015;35(3):236-244. doi:10.1111/neup.12187

8. Sun H, Yin L, Li S, et al. Prognostic significance of IDH mutation in adult low-grade gliomas: a meta-analysis. J Neurooncol. 2013;113 (2):277-284. doi:10.1007/s11060-013-1107-5

9. Wang L, Li Z, Liu C, et al. Comparative assessment of three methods to analyze MGMT methylation status in a series of 350 gliomas and gangliogliomas. Pathol Res Pract. 2017;213(12):1489-1493. doi:10.1016/j.prp.2017.10.007

10. Wuhao H, Shengguang W, Hua Z, Bin Z, Changli W. Prognostic significance of combined fibrinogen concentration and neutrophil-to-lymphocyte ratio in patients with resectable non-small cell lung cancer. Cancer Biol Med. 2018;15(1):88. doi:10.20892/j.issn.2095-3941.2017.0124

11. Tu XP, Qiu QH, Chen LS, et al. Preoperative neutrophil-to-lymphocyte ratio is an independent prognostic marker in patients with laryngeal squamous cell carcinoma. BMC Cancer. 2015;15:743. doi:10.1186/ s12885-015-1727-6

12. Templeton AJ, McNamara MG, Seruga B, et al. Prognostic role of neutrophil-to-lymphocyte ratio in solid tumors: a systematic review and meta-analysis. $J$ Natl Cancer Inst. 2014;106(6):dju124. doi:10.1093/jnci/dju124

13. McNamara MG, Lwin Z, Jiang $\mathrm{H}$, et al. Factors impacting survival following second surgery in patients with glioblastoma in the temozolomide treatment era, incorporating neutrophil/lymphocyte ratio and time to first progression. $J$ Neurooncol. 2014;117(1):147-152. doi:10.1007/s11060-014-1366-9

14. Fujii $M$, Yamashita $T$, Ishiguro $R$, Tashiro $M$, Kameyama $K$. Significance of epidermal growth factor receptor and tumor associated tissue eosinophilia in the prognosis of patients with nasopharyngeal carcinoma. Auris Nasus Larynx. 2002;29:175-181. doi:10.1016/ S0385-8146(01)00135-3 
15. Dorta RG, Landman G, Kowalski LP, Lauris JR, Latorre MR, Oliveira DT. Tumour-associated tissue eosinophilia as a prognostic factor in oral squamous cell carcinomas. Histopathology. 2002;41 (2):152-157. doi:10.1046/j.1365-2559.2002.01437.x

16. Arigami T, Uenosono Y, Ishigami S, et al. A novel scoring system based on fibrinogen and the neutrophil-lymphocyte ratio as a predictor of chemotherapy response and prognosis in patients with advanced gastric cancer. Oncology. 2016;90(4):186-192. doi:10.1159/000444494

17. Arigami T, Okumura H, Matsumoto M, et al. Analysis of the fibrinogen and neutrophil-lymphocyte ratio in esophageal squamous cell carcinoma: a promising blood marker of tumor progression and prognosis. Medicine. 2015;94(42):e1702. doi:10.1097/MD.0000000000001702

18. Davis BP, Rothenberg ME. Eosinophils and cancer. Cancer Immunol Res. 2014;2(1):1-8. doi:10.1158/2326-6066.CIR-13-0196

19. Kaur H, Lachance DH, Ryan CS, et al. Asthma and risk of glioma: a population-based case-control study. BMJ Open. 2019;9(6):e025746. doi:10.1136/bmjopen-2018-025746

20. Lachance DH, Yang P, Johnson DR, et al. Associations of high-grade glioma with glioma risk alleles and histories of allergy and smoking. Am J Epidemiol. 2011;174(5):574-581. doi:10.1093/aje/kwr124

21. Il'yasova D, McCarthy B, Marcello J, et al. Association between glioma and history of allergies, asthma, and eczema: a case-control study with three groups of controls. Cancer Epidemiol Biomarkers Prev. 2009;18(4):1232-1238. doi:10.1158/1055-9965.EPI-08-0995

22. Caruso RA, Parisi A, Quattrocchi E, et al. Ultrastructural descriptions of heterotypic aggregation between eosinophils and tumor cells in human gastric carcinomas. Ultrastruct Pathol. 2011;35(4):145-149. doi: $10.3109 / 01913123.2011 .578233$

23. Simson L, Ellyard JI, Dent LA, et al. Regulation of carcinogenesis by IL-5 and CCL11: a potential role for eosinophils in tumor immune surveillance. J Immunol. 2007;178(7):4222-4229. doi:10.4049/ jimmunol.178.7.4222

24. Huang Z, Wu L, Hou Z, Zhang P, Li G, Xie J. Eosinophils and other peripheral blood biomarkers in glioma grading: a preliminary study. BMC Neurol. 2019;19(1):313. doi:10.1186/s12883-019-1549-2

25. Rachidi S, Wallace K, Wrangle JM, Day TA, Alberg AJ, Li Z. Neutrophil-to-lymphocyte ratio and overall survival in all sites of head and neck squamous cell carcinoma. Head Neck. 2016;38(S1): E1068-E1074. doi:10.1002/hed.24159

26. He JR, Shen GP, Ren ZF, et al. Pretreatment levels of peripheral neutrophils and lymphocytes as independent prognostic factors in patients with nasopharyngeal carcinoma. Head Neck. 2012;34 (12):1769-1776. doi:10.1002/hed.22008
27. Jablonska J, Leschner S, Westphal K, Lienenklaus S, Weiss S. Neutrophils responsive to endogenous IFN- $\beta$ regulate tumor angiogenesis and growth in a mouse tumor model. $J$ Clin Investig. 2010;120(4):1151-1164. doi:10.1172/JCI37223

28. Galon J, Costes A, Sanchez-Cabo F, et al. Type, density, and location of immune cells within human colorectal tumors predict clinical outcome. Science. 2006;313(5795):1960-1964. doi:10.1126/science.1129139

29. Loi S, Sirtaine N, Piette F, et al. Prognostic and predictive value of tumor-infiltrating lymphocytes in a Phase III randomized adjuvant breast cancer trial in node-positive breast cancer comparing the addition of docetaxel to doxorubicin with doxorubicin-based chemotherapy: BIG 02-98. J Clin Oncol. 2013;31(7):860-867. doi:10.1200/JCO.2011.41.0902

30. Zheng SH, Huang JL, Chen M, Wang BL, Ou QS, Huang SY. Diagnostic value of preoperative inflammatory markers in patients with glioma: a multicenter cohort study. J Neurosurg. 2018;129 (3):583-592. doi:10.3171/2017.3.JNS161648

31. Wang Z, Zhong L, Li G, et al. Pre-treatment neutrophils count as a prognostic marker to predict chemotherapeutic response and survival outcomes in glioma: a single-center analysis of 288 cases. $\mathrm{Am}$ J Transl Res. 2020;12(1):90.

32. Balachandran VP, Gonen M, Smith JJ, DeMatteo RP. Nomograms in oncology: more than meets the eye. Lancet Oncol. 2015;16(4):e173e180. doi:10.1016/S1470-2045(14)71116-7

33. Iasonos A, Schrag D, Raj GV, Panageas KS. How to build and interpret a nomogram for cancer prognosis. J Clin Oncol. 2008;26 (8):1364-1370. doi:10.1200/JCO.2007.12.9791

34. Montero PH, Yu C, Palmer FL, et al. Nomograms for preoperative prediction of prognosis in patients with oral cavity squamous cell carcinoma. Cancer. 2014;120(2):214-221. doi:10.1002/cncr.28407

35. Li Y, Jia H, Yu W, et al. Nomograms for predicting prognostic value of inflammatory biomarkers in colorectal cancer patients after radical resection. Int J Cancer. 2016;139(1):220-231. doi:10.1002/ijc.30071

36. Gittleman H, Sloan AE, Barnholtz-Sloan JS. An independently validated survival nomogram for lower grade glioma. Neuro Oncol. 2019.

37. Zhao YY, Chen SH, Hao Z, Zhu HX, Xing ZL, Li MH. A nomogram for predicting individual prognosis of patients with low-grade glioma. World Neurosurg. 2019;130:e605-e612. doi:10.1016/j.wneu.2019.06.169

38. Guthrie GJ, Charles KA, Roxburgh CS, Horgan PG, McMillan DC, Clarke SJ. The systemic inflammation-based neutrophil-lymphocyte ratio: experience in patients with cancer. Crit Rev Oncol Hematol. 2013;88(1):218-230. doi:10.1016/j.critrevonc.2013.03.010

\section{Publish your work in this journal}

Cancer Management and Research is an international, peer-reviewed open access journal focusing on cancer research and the optimal use of preventative and integrated treatment interventions to achieve improved outcomes, enhanced survival and quality of life for the cancer patient.
The manuscript management system is completely online and includes a very quick and fair peer-review system, which is all easy to use. Visit http://www.dovepress.com/testimonials.php to read real quotes from published authors. 